\title{
Fish Egg Poisoning: An Unusual Cause of Respiratory Paralysis
}

Sir,

A 10-yr-old boy was brought with complaints of recurrent bouts of vomiting, pain abdomen and paraesthesias around his lips within an hr of ingesting freshly cooked fish eggs. Later over the next two hr he developed unsteadiness and difficulty in walking and standing. He was treated with intravenous fluids and kept under observation in Emergency Medical Services unit of our hospital. His blood pressure, heart rate and respiratory rate were normal with features of mild dehydration. His serum electrolytes were normal . In the hospital, over the next four hours, he became drowsy and dysarthric with weakness of all four limbs with areflexia and difficulty in swallowing and abnormal movements of tongue and limbs. He was unable to speak. He developed drooling of secretions and choking after taking sips of water and subsequently developed difficulty in breathing and paradoxical breathing. The child received ventilatory support with minimal settings and was gradually weaned and extubated over the next $28 \mathrm{hr}$. He was on intravenous fluids, injection ceftriaxone, injection metronidazole and ranitidine. His weakness gradually improved over the next $24 \mathrm{hr}$ and was able to sit up and talk and eat and drink. Over the next $48 \mathrm{hr}$ he completely recovered and there were no focal neurological deficits. Nerve conduction study showed decreased CMAP amplitudes and motor nerve conduction velocities of right ulnar and common peroneal nerve.

Reports of paralysis resulting from ingestion of puffer fish or its eggs from Southeast Asia have been documented in literature. ${ }^{1,2}$ The toxin incriminated in puffer fish poisoning includes saxitoxin and tetradotoxin, which inhibit the voltage gated $\mathrm{Na}$ channels with axonal conduction blocks causing paralysis of skeletal muscle and diaphragm. The symptoms of puffer fish poisoning typically start within half an hr of ingestion of toxin containing fish and include vomiting, pain abdomen, perioral parasthesias and respiratory paralysis within 4-6 $\mathrm{hr} .^{3}$ These toxins are produced by marine dinoflagellates and the fish acquire them by feeding on these dinoflagellates. The toxin producing algae and diatoms are found in abundance during a phenomenon referred to as the red tide which is nothing but the discolouration of ocean waters by the algal bloom - referred to as the harmful algal bloom. The centre for marine living resources and ecology under the Ministry of Earth Sciences Kochi, Kerala has implemented a programme for monitoring and surveillance of harmful algal bloom in and around the Indian exclusive economic zone and has documented close to 400 species of toxic algae. ${ }^{4}$

The clinical picture in this child mimics botulism and Guillain Barre Syndrome. The rapid onset of symptoms and the rapid progression to respiratory paralysis, quick recovery following supportive care and the absence of ophthalmoplegia and pupillary signs in this boy suggest a clinical diagnosis of puffer fish poisoning. Similar symptoms had also been observed in individuals with shell fish poisoning in Tamil Nadu in 1981 and in Mangalore in $1983 .{ }^{5}$

Eating exotic varieties of fish may prove fatal especially during the presence of red tide or natural catastrophes like tsunami. Children brought with sea food ingestion and vomiting should always be closely observed for a minimum of 6 to $8 \mathrm{hr}$ for evolution of neurological symptoms before they can be discharged.

Venkatesh Chandrasekaran, Sriram Pothapregada and Mahadevan Subramanian Department of Pediatrics, JIPMER, Ponducherry-6, India E-mail: cvenkatesh@hotmail.com [DOI-10.1007/s12098-009-0304-x]

\section{REFERENCES}

1. Kanchanapongkul J. Puffer fish poisoning: clinical features and management experience in 25 cases. J Med Assoc Thai 2001; 84: 385-389.

2. Kan SK, Chan MK, David P. Nine fatal cases of puffer fish poisoning in Sabah, Malaysia. Med J Malaysia 1987; 42: 199200.

3. Centres for Disease Control and Prevention. Diagnosis and management of Food borne Illnesses: A Primer for Physicians and Other Healthcare Professionals. MMWR Recommendations and reports 2004; 53: 11-12.

4. http://cmlre.gov.in/hc.html.

5. Bhat SR, Prabhu Matondkar SG.Algal blooms in the seas around India-Networking for research and outreach. Current science 2004; 87: 1081. 